\title{
A Discrete Ordinates Solution of the Fokker-Planck Equation Characterizing Charged Particle Transport*
}

\author{
Thomas A. Mehlhorn ${ }^{\dagger}$ and James J. Duderstadt \\ Department of Nuclear Engineering, The University of Michigan \\ Ann Arbor, Michigan 48109
}

Received April 25, 1979; revised December 10, 1979

\begin{abstract}
A formalism for obtaining a discrete ordinates solution of the time and space-dependent Fokker-Planck equation governing the transport of charged particles in multispecie plasmas is developed. In the absence of macroscopic electromagnetic fields and assuming isotropic Rosenbluth potentials, the Fokker-Planck equation is solved for a test particle distribution; both angular dispersion and velocity diffusion are accounted for. Difference relations are obtained and a series of validation problems are discussed. The conservation of both particles and energy are continuously monitored. In addition to providing the single-particle distribution function $f(r, \mu, v, t)$, spatially dependent energy deposition profiles are calculated. Comparisons with reported energy deposition profiles for a central source of $3.5-\mathrm{MeV}$ alpha particles in a spherical D-T plasma are made and are found to be in good agreement.
\end{abstract}

\section{INTRODUCTION}

The accurate simulation of the transport of energetic (i.e., superthermal) charged particles is of vital importance to the success of many controlled thermonuclear fusion schemes. For example, there has been great interest in simulating the transport of superthermal electrons during the implosion process in inertial confinement schemes (notably laser fusion) as well as investigating burn-wave propagation via the deposition of the $3.5-\mathrm{MeV}$ alpha particle energy in the dense core of a compressed microsphere. In inertial confinement schemes using either electrons or ion beams, there are many questions to be studied concerning the deposition of the charged particle energy in the target, the propagation of beams through space-charge neutralizing media, and the like, that likewise require an accurate simulation of energetic charged particle transport. Charged particle transport also plays a fundamental role in magnetic fusion schemes. For example, there is considerable interest in obtaining solutions of the spatially inhomogeneous Fokker-Planck equation to study the energy deposition from neutral beam injection into Tokamak devices where the ionized beam particles are either directly injected or scattered into trapped orbits.

* Work supported by the Air Office of Scientific Research.

${ }^{+}$Permanent address: Sandia Laboratories, Division 4247, Albuquerque, N. M. 87185. 
The interaction mechanism of most concern in the transport of energetic charged particles in plasmas involves small-angle scattering collisions. Large-angle scattering occurs much less frequently and will not be treated in this work. The most common description of the cumulative effect of many random, small-angle collisions on a distribution of particles is provided by the Fokker-Planck equation. Since the Fokker-Planck equation is commonly used as the basis for most numerical studies of charged particle transport in plasmas, a variety of methods have been developed to solve it. In much of the early work such as that developed for modeling magnetic mirror devices [1], the spatial dependence was only included via a bounce-average approximation. More recent methods which specifically account for spatial effects include the modified multigroup flux-limited diffusion scheme of Corman et al. |2|; the LSN treatment of the equations of mass and energy transport by Antal and Lee $|3|$, the modified moment method of Haldy and Ligou $[4]$, and the integral particle tracking techniques of Moses [5]. However, most of these schemes involve the introduction of substantial approximations into the Fokker-Planck equation in order to arrive at a set of equations that are amenable to numerical solutions.

In this paper we describe a direct numerical approach which solves a more general form of the time-, space-, and velocity-dependent Fokker-Planck equation. In order to facilitate future generalizations of our Fokker-Planck collision term physics we have utilized the formalism of Killeen and Marx [1] which results in a common notation between this work and others containing more sophisticated velocity-space physics (e.g., see [6]). The discrete ordinate method has been used since it is a highly developed numerical technique for obtaining deterministic solutions to the transport equation.

The use of the discrete ordinate method [7] for solving the spatially dependent Fokker-Planck equation is particularly attractive since it has been highly developed for the solution of radiation transport problems. Indeed, there are a variety of sophisticated and efficient one- and two-dimensional computer codes available that have planar, cylindrical, and spherical geometry options (as well as toroidal [8] and triangular meshes [9] for nonorthogonal geometries) and allow for a variety of boundary and source conditions. Therefore, the successful incorporation of the Fokker-Planck collision term into the multigroup discrete ordinates formalism will provide for an easy extension to a variety of geometries and source configurations.

In Section 2 we will develop the particular form of the Fokker-Planck equation that we have analyzed. We then introduce the numerical techniques that we have employed to difference the Fokker-Planck collision term in a manner that is compatible with the formalism of a standard multigroup discrete ordinates code in Section 3. This analysis has been implemented by modifying the TIMEX [10] timedependent discrete ordinates computer code to include charged particle collisions as described by the Fokker-Planck collision term. We refer to this modified code, naturally enough, as TIMEX-FP. Section 4 summarizes the procedure we used to validate the code. It is felt that the retention of certain physical terms (e.g., the velocity dispersion term and general slowing down physics) has facilitated the validation of the TIMEX-FP code. The results of a benchmark calculation describing 
fusion reaction product energy deposition in spherical geometry are presented in Section 5 .

\section{Basic Formalism}

We begin with a general form of the equation characterizing particle transport:

$$
\frac{\partial f}{\partial t}+\mathbf{v} \cdot \frac{\partial f}{\partial \mathbf{r}}+\frac{F}{m} \cdot \frac{\partial f}{\partial \mathbf{v}}=\left.\frac{\partial f}{\partial t}\right|_{\text {collisions }}+S
$$

Our first task is to cast this equation with a Fokker-Planck collision term into a form suitable for analysis using multigroup discrete ordinate methods. Rosenbluth et al. $|11|$ have written the Fokker-Planck collision term

$$
\left.\frac{1}{\Gamma_{T}} \frac{\partial f_{T}}{\partial t}\right|_{\text {coll }}=-\frac{\partial}{\partial \mathbf{v}} \cdot\left(f_{T} \frac{\partial h_{T}}{\partial \mathbf{v}}\right)+\frac{1}{2} \frac{\partial^{2}}{\partial \mathbf{v} \partial \mathbf{v}}:\left(f_{T} \frac{\partial^{2} g_{T}}{\partial \mathbf{v} \partial \mathbf{v}}\right),
$$

where the so-called Rosenbluth potentials are given by

$$
\begin{aligned}
& h_{T}=\sum_{b}\left(1+\frac{m_{T}}{m_{b}}\right)\left(\frac{z_{b}}{z_{T}}\right)^{2} \int d \mathbf{v}^{\prime} f_{b}\left(\mathbf{v}^{\prime}, t\right)\left|\mathbf{v}-\mathbf{v}^{\prime}\right|^{-1}, \\
& g_{T}=\sum_{b}\left(\frac{z_{b}}{z_{T}}\right)^{2} \int d \mathbf{v}^{\prime} f_{b}\left(\mathbf{v}^{\prime}, t\right)\left|\underline{v}-\underline{v}^{\prime}\right| .
\end{aligned}
$$

The distribution function $f_{T}(\mathbf{r}, \mathbf{v}, \mathbf{t})$ has its usual significance where the subscript $T$ refers to the test particle and the subscript $b$ refers to the various background particles (ions or electrons). Following Killeen and Marx [1], we then choose an azimuthally symmetric spherical-polar coordinate system in velocity space and make the assumption that the Rosenbluth potentials are isotropic. The transformation of Eqs. (2) and (3) into such a system leads to

$$
\begin{aligned}
\left.\frac{1}{\Gamma_{T}} \frac{\partial f_{T}}{\partial t}\right|_{\text {coll }}= & -\frac{1}{v^{2}} \frac{\partial}{\partial v}\left[f_{T}\left(v^{2} \frac{\partial h_{T}}{\partial v}+\frac{\partial g_{T}}{\partial v}\right)\right]+\frac{1}{2 v^{2}} \frac{\partial^{2}}{\partial v^{2}}\left[v^{2} f_{T} \frac{\partial^{2} g_{T}}{\partial v^{2}}\right] \\
& +\frac{1}{2 v^{3}} \frac{\partial g_{T}}{\partial v}\left[\frac{\partial}{\partial \mu}\left[\left(1-\mu^{2}\right) \frac{\partial f_{T}}{\partial \mu}\right]\right] \\
h_{T}= & 4 \pi \sum_{b}\left(1+\frac{m_{T}}{m_{b}}\right)\left(\frac{z_{b}}{z_{T}}\right)^{2}\left[\int_{0}^{t} f_{b}\left(v^{\prime}, t\right) \frac{v^{\prime 2}}{v} d v^{\prime}+\int_{v}^{\infty} f_{b}\left(v^{\prime}, t\right) v^{\prime} d v^{\prime}\right] \\
g_{T}= & 4 \pi \sum_{b}\left(\frac{z_{b}}{z_{T}}\right)^{2}\left[\int_{0}^{v} f_{b}\left(v^{\prime}, t\right) v\left(1+\frac{1}{3} \frac{v^{\prime 2}}{v^{2}}\right) v^{\prime 2} d v^{\prime}\right. \\
& \left.+\int_{v}^{\infty} f_{b}\left(v^{\prime}, t\right)\left(1+\frac{1}{3} \frac{v^{2}}{v^{\prime 2}}\right) v^{\prime 3} d v^{\prime}\right]
\end{aligned}
$$


At this point we introduce the dimensionless parameter $x=v / v_{0}$ where $v_{0}$ is some constant reference velocity, and define the following standard integrals:

$$
\begin{aligned}
& N\left(f_{b}\right)=\int_{0}^{x} f_{b}(y, t) y^{2} d y, \\
& M\left(f_{b}\right)=\int_{x}^{\infty} f_{b}(y, t) y d y, \\
& E\left(f_{b}\right)=\int_{0}^{x} f_{b}(y, t) y^{4} d y .
\end{aligned}
$$

By evaluating the derivatives of the Rosenbluth potentials (Eq. (5)) that occur in Eq. (4) using the Leibnitz formula for the derivative of an integral, we can rewrite the collision term in terms of these integrals as

$$
\left.\frac{v_{0}^{3}}{\Gamma_{T}} \frac{\partial f_{T}}{\partial t}\right|_{\text {coll }}=\frac{1}{x^{2}} \frac{\partial}{\partial x}\left[A f_{T}+B \frac{\partial f_{T}}{\partial x}\right]+\frac{C}{2 x^{3}} \frac{\partial}{\partial \mu}\left[\left(1-\mu^{2}\right) \frac{\partial f_{T}}{\partial \mu}\right],
$$

where

$$
\begin{aligned}
& A=\sum_{b}\left(\frac{z_{b}}{z_{T}}\right)^{2}\left(\frac{m_{T}}{m_{b}}\right) N\left(f_{b}\right), \\
& B=\sum_{b}\left(\frac{z_{b}}{z_{T}}\right)^{2}\left\{\frac{1}{3 x} E\left(f_{b}\right)+\frac{x^{2}}{3} M\left(f_{b}\right)\right\}, \\
& C=\sum_{b}\left(\frac{z_{b}}{z_{T}}\right)^{2}\left\{N\left(f_{b}\right)-\frac{1}{3 x^{2}} E\left(f_{b}\right)+\frac{2 x}{3} M\left(f_{b}\right)\right\} .
\end{aligned}
$$

Note that $\Gamma_{T}=\left(4 \pi z_{T}^{4} e^{4} n_{b} / m_{T}^{2}\right) \ln A_{b}$, where $z, m, n$, and $\ln A_{b}$ are respectively the atomic number, mass, particle density, and Coulomb logarithm for plasma specie $b$. The variable $\mu$ is defined as the cosine of the angle between the velocity vector $\mathbf{v}$ and the position vector $\mathbf{r}$.

At this point we will restrict our analysis by assuming that the velocity distributions for the various background plasma components can be given by a Maxwellian of the form:

$$
f_{b}=\frac{4}{\pi^{1 / 2}}\left(\frac{v_{0}}{v_{b}}\right)^{3} e^{-\left(v_{0} / v_{b}\right)^{2} x^{2}}
$$

where $v_{b}$ is the thermal velocity of the $b$ th plasma component. This assumption linearizes the equation by excluding the nonlinear test particle-test particle interactions (self-collisions) and does not allow for a consistent treatment of the coupled relaxation of the two interacting plasma components (namely, the test and field particles). This model is consistent with the macroscopic information, i.e., 
density and temperature profiles, available from a hydrodynamic code. It should be noted that since we have carefully defined our coefficients (Eq. (8)) in terms of the standard integrals (Eq. (6)), it is possible to relax this assumption if it is deemed necessary by numerically evaluating the integrals in a consistent fashion using the detailed distribution function in an iterative fashion; however, rigorously this would require the inclusion of anisotropic Rosenbluth potentials into the formalism.

Now, substituting our assumed velocity distribution into the standard integrals and performing the integrations, we find

$$
\begin{aligned}
& A=\sum_{b}\left(\frac{z_{b}}{z_{T}}\right)^{2} \rho_{b}^{2} G\left(\gamma_{b}^{1 / 2} x\right), \\
& B=\sum_{b}\left(\frac{z_{b}}{z_{T}}\right)^{2} \gamma_{b}^{-1} G\left(\gamma_{b}^{1 / 2} x\right) \frac{1}{2 x}, \\
& C=\sum_{b}\left(\frac{z_{b}}{z_{T}}\right)^{2} L\left(\gamma_{b}^{1 / 2} x\right),
\end{aligned}
$$

where

$$
\begin{aligned}
G(y) & =\phi(y)-\frac{2 y}{\pi^{1 / 2}} e^{-y^{2}}, \\
L(y) & =\left(1-\frac{1}{2 y^{2}}\right) G(y)+\frac{2 y}{\pi^{1 / 2}} e^{-y^{2}} \\
\rho_{b}^{2} & =\frac{m_{T}}{m_{b}}, \\
\gamma_{b} & =\left(\frac{v_{0}}{v_{b}}\right)^{2} . \\
\phi(y) & =\operatorname{erf}(y) .
\end{aligned}
$$

We complete the specification of our coefficients by choosing the reference velocity $v_{0}$ to be the velocity that the test particle would have when in thermal equilibrium with the background plasma,

$$
v_{0} \equiv\left(\frac{2 \theta_{b}}{m_{r}}\right)^{1 / 2}
$$

where $\theta_{b}$ is the temperature of the background plasma component $b$. Note that we will invoke the simplifying assumption that all the background plasma species have the same temperature although the analysis does not require it. Using this we find that $\gamma_{b} \equiv \rho_{b}^{-2}$, such that 


$$
\begin{gathered}
A(x)=2 x B(x)=\sum_{b}\left(\frac{z_{b}}{z_{T}}\right)^{2} \rho_{b}^{2} G\left(x / \rho_{b}\right), \\
C(x)=\sum_{b}\left(\frac{z_{b}}{z_{T}}\right)^{2} L\left(x / \rho_{b}\right) .
\end{gathered}
$$

These coefficients, along with the following equation, define the numerical system that we wish to investigate.

$$
\frac{\partial f}{\partial \tau}+\mathbf{x} \cdot \frac{\partial f}{\partial \xi}=\frac{1}{x^{2}} \frac{\partial}{\partial x}\left\{A(x) f+\frac{A(x)}{2 x} \frac{\partial f}{\partial x}\right\}+\frac{C(x)}{2 x^{3}} \frac{\partial}{\partial \mu}\left[\left(1-\mu^{2}\right) \frac{\partial f}{\partial \mu}\right]+S t_{0},
$$

where

$$
\begin{array}{ll}
\tau=\frac{t}{t_{0}}, & t_{0}=\frac{v_{0}^{3}}{\Gamma_{T}} \\
\xi=\frac{r}{r_{0}}, & r_{0}=\frac{v_{0}^{4}}{\Gamma_{T}}
\end{array}
$$

Here we have also chosen to ignore the macroscopic force term $(\mathbf{F} / m) \cdot(\partial f / \partial \mathbf{v})$, and therefore the effects of both external as well as self-consistent fields on particle transport will not be included in this analysis. Moreover, an external source term, $S t_{0}$, has been included for completeness.

\section{Numerical Analysis}

Since the Fokker-Planck collision term as defined in Eq. (14) has distinct terms for scattering with energy transfer and scattering with angular deflection, we have chosen to perform the development and validation of suitable difference methods for each of these terms separately. This has allowed us to compare the results of our analysis with simple analytical estimates.

We have chosen to implement our numerical procedure for solving the Fokker-Planck equation by modifying a versatile time-dependent discrete ordinates code, TIMEX, to allow for the Fokker-Planck collision term. TIMEX is a timedependent particle transport code originally developed for the study of neutron transport. It utilizes a discrete ordinates treatment of the particle direction (angle), a discontinuous, linear finite-element treatment of the spatial variable, a multigroup treatment of the energy variable, and an explicit time differencing scheme.

The term "multigroup" in neutron transport theory refers to partitioning of the energy domain into a set of intervals of width $\Delta E_{g}$ such that

$$
\psi_{g}=\int_{\Delta E_{g}} \psi d E
$$


Here $\psi$ is the so-called "flux" and is given by the product of the particle speed $v$ and the distribution function $f$. By convention an increasing index $g$ represents decreasing energy. In TIMEX, the relevant terms of the neutron transport equation take the following form after multigrouping in energy and explicitly differencing the time variable:

$$
\begin{aligned}
\frac{1}{v \Delta t}\left(\psi_{g}^{n+1}-\psi_{g}^{n}\right)+\nabla \cdot\left(\Omega \psi_{g}^{n+1}\right)+\sigma_{g} \psi_{g}^{n+1}(r, \Omega) \\
=\sum_{h=1}^{g-1} \sigma_{s, h \rightarrow R} \psi_{h}^{n+1}(r)+\sum_{h=R}^{G} \sigma_{s, h \rightarrow R} \psi_{h}^{n}(r)+S_{R}^{n+1}(r),
\end{aligned}
$$

where

$$
\Omega=\underline{v} /|\underline{v}|, \quad \psi^{n}=\psi\left(t^{n}\right) .
$$

Here, $\sigma_{g}$ represents the total cross section for the loss of particles from group $g$, the summation up to group $g-1$ represents downscattering of particles from higherenergy groups, while the summation up to the maximum group $G$ represents up and self-scattering. $S_{g}$ is the group source term. The arrangement of the differenced angular flux for the $i, m$ (space, angle) mesh cell is illustrated in Fig. 1.

The system of equations for the mesh cell edge fluxes in TIMEX takes the form

$$
\overline{\bar{A}}\left[\begin{array}{l}
\psi_{i-1 / 2} \\
\psi_{i+1 / 2}
\end{array}\right]=\left[\begin{array}{l}
S_{i-1 / 2} \\
S_{i+1 / 2}
\end{array}\right] .
$$

This system of equations is then solved along all discrete angular rays $\mu_{m}$, first for $\mu_{m}>0$, then for $\mu_{m}<0$ (this defines a space-angle sweep) and a space-angle sweep is performed for each energy group beginning with the highest group $g=1$. These operations comprise one time step.

The suitability of these features for the analysis of charged particle transport will become apparent as we proceed with our development. We refer to the modified version of this code as TIMEX-FP.

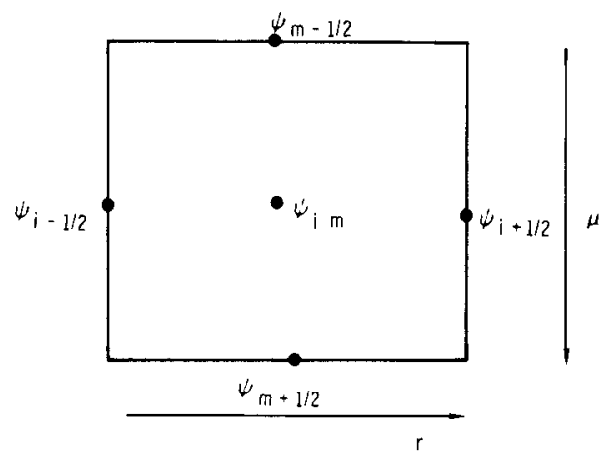

FIGURE 1 


\subsection{Angular Dispersion Term}

First, consider the angular dispersion term which takes the form

$$
\gamma \frac{\partial}{\partial \mu}\left[\left(1 \cdots \mu^{2}\right) \frac{\partial f}{\partial \mu}\right]
$$

For our initial studies, we chose to difference this term following the so-called " $\alpha$ coefficient" method often used for discretizing the spatial divergence term in spherical geometry:

$$
\frac{\partial}{\partial \mu}\left[\left(1-\mu^{2}\right) \frac{\partial f}{\partial \mu}\right] \rightarrow \frac{1}{\omega_{m}}\left[\left.\left(1-\mu_{m+1 / 2}^{2}\right) \frac{\partial f}{\partial \mu}\right|_{m+1 / 2}-\left.\left(1-\mu_{m-1 / 2}^{2}\right) \frac{\partial f}{\partial \mu}\right|_{m-1 / 2}\right] .
$$

The derivative term in Eq. (19) is simply replaced by a centered difference expression to yield

$$
\left.\frac{\partial f}{\partial \mu}\right|_{m+1 / 2}=\frac{f_{m+1}-f_{m}}{\Delta \mu_{m+1 / 2}}
$$

with

$$
\begin{aligned}
\Delta \mu_{m+1 / 2} & =\mu_{m+1}-\mu_{m}, \\
\mu_{m+1 / 2} & =\frac{1}{2}\left(\mu_{m+1}+\mu_{m}\right) .
\end{aligned}
$$

Here, $\omega_{m}$ refers to the quadrature weight associated with the $m$ th angular quadrature point $\mu_{m}$. Note that $f_{m}$ is really $f_{m}^{i}$ (the cell-centered value), where $i$ is the spatial index. This cell-centered value is computed using the diamond difference relations from the known cell-edge values.

$$
f_{m}^{i}=\frac{1}{2}\left[f_{m}^{i+1 / 2}+f_{m}^{i-1 / 2}\right] \text {. }
$$

If this term is to preserve the conservation of particles when integrated over the angular domain, we must require that the quadrature of the difference relation sum to zero. The resulting conservation requirement takes the form

$$
\mu_{1 / 2}=-1, \quad \mu_{\mathrm{MM}+1 / 2}=1
$$

where $\mathrm{MM}$ is the maximum angular index as determined by the order of the angular quadrature, $S_{N}$.

Since this term does not directly correspond to any term in the standard multigroup transport formalism, we concluded that it would be most advantageous and natural to include this angular redistribution term as an effective source term in our discrete ordinates code of the form

$$
\overline{\bar{A} f}=\mathbf{S}=\left[\begin{array}{c}
S_{1}^{\prime} \\
S_{2}^{\prime}
\end{array}\right]=\left[\begin{array}{c}
S_{1}+C_{1} \gamma / \omega_{m}\{\ldots\} \\
S_{2}+C_{2} \psi / \omega_{m}\{\ldots\}
\end{array}\right]
$$


TABLE I

Spatial Geometric Coefficients for the Angular Dispersion Term

\begin{tabular}{llc}
\hline Coefficient & \multicolumn{2}{c}{ Geometry } \\
\cline { 2 - 3 }$C_{1}$ & Planar & Spherical \\
$C_{2}(\mu>0)$ & $\Delta r_{i} V_{i}^{c}$ & $\frac{r_{+}^{a}}{3} A_{+}^{n}-\frac{r_{-}^{a}}{3} A^{b}$ \\
$C_{2}(\mu<0)$ & $\Delta r_{i} V_{i}$ & $\Delta r_{i} \frac{\pi}{3}\left[3 r_{+}^{2}+2 r_{+} r_{-}+r_{-}^{2} \mid\right.$ \\
\hline
\end{tabular}

$$
\begin{array}{ll}
a r_{+}=r_{i+1 / 2} & r_{-}=r_{i-1 / 2} \\
b A_{+}=4 \pi r_{+}^{2} & A_{-}=4 \pi r_{-}^{2} \\
\text { c } V=\frac{1}{2} \Delta r &
\end{array}
$$

Here, $C_{1}$ and $C_{2}$ are the appropriate spatial geometric coefficients necessary to make the scattering term compatible with the discontinuous spatial finite element treatment found in the TIMEX code. The geometric coefficients have been calculated and are contained in Table I.

Finally, the angular dispersion term was differenced in a manner that was compatible with the time differencing scheme in TIMEX. The second-order derivative in angle associated with this term results in a temporal coupling of the space-angle discrete ordinates equation when one uses a forward time differencing scheme such as that found in TIMEX. In analogy to the case of up and self-scattering in the multigroup formalism of transport theory, we have chosen to break this coupling by treating the unknown values of the distribution function on the angular mesh in an explicit manner. That is, the derivatives take the form

$$
\left.\frac{\partial f}{\partial \mu}\right|_{m+1 / 2} ^{n+1} \cong \frac{f_{m+1}^{n}-f_{m}^{n}}{\Delta \mu_{m+1 / 2}},\left.\quad \frac{\partial f}{\partial \mu}\right|_{m-1 / 2} ^{n+1} \cong \frac{f_{m}^{n}-f_{m-1}^{n+1}}{\Delta \mu_{m-1 / 2}}
$$

where the index $n$ refers to the time index $t^{n}$. Note that the value $f_{m-1}$ is taken from the $(n+1)$ st time step in accordance with the explicit time differencing of the neutron transport equation (cf. Eq. (16)). (The explicit differencing inherent in the TIMEX code results in the continual updating of the angular flux storage vectors and the overwriting of the values $f_{m-1}^{n}$.) The explicit nature of the calculation means that the accuracy of the final solution is dependent upon the requirement that the time-step 
size be small such that the value of $f$ at time $n+1$ does not change greatly from that of time $n$. This restriction on the time-step size is also required to preserve the conservation property of the angular dispersion term, although we have found that the requirements to insure the positivity of the solution, as noted below, are more stringent than those necessary to insure conservation of particles.

\subsection{Drag and Velocity Diffusion Terms}

We have been able to discretize the drag and velocity diffusion terms in a form that is compatible with the multigroup formalism of neutron transport theory. In order to simplify the following discussion we will restrict our attention to the isotropic velocity space form of the Fokker-Planck equation as given by

$$
\frac{\partial f}{\partial t}=\frac{1}{x^{2}} \frac{\partial}{\partial x}\left\{A(x) f+\frac{A(x)}{2 x} \frac{\partial f}{\partial x}\right\}
$$

and where the definition of the various terms and coefficients remain as previously defined.

Preliminary attempts to apply the multigroup technique directly to this term yielded some unsatisfactory results. This occured because the direct application of the multigroup technique requires the specification of an additional relation between the within-group distribution function and the distribution function at a group boundary, (e.g., see Ref. [2] and refer to the definition of the multigroup flux in Eq. (15)). Therefore, we decided to investigate the use of a pointwise energy differencing (i.e., $\psi_{g}=\psi\left(E_{g}\right)$ ) rather than the traditional multigroup formalism. To facilitate matters, we chose to adapt the differencing scheme introduced by Chang and Cooper [12] to arrive at a system of equations that is algebraically equivalent to those obtained using the standard multigroup formalism.

The differenced form of Eq. (26) is

$$
\frac{1}{\Delta \tau}\left[f_{j}^{n+1}-f_{j}^{n}\right]=\frac{1}{x_{j}^{2} \Delta x_{j}}\left\{P_{j} f_{j+1}^{n+1}-Q_{j} f_{j}^{n+1}+R_{j} f_{j-1}^{n+1}\right\}
$$

where

$$
\begin{aligned}
& P_{j}=A\left(x_{j+1 / 2}\right) W_{j} e^{\omega_{j}} / \Delta x_{j+1 / 2}, \\
& R_{j}=A\left(x_{j-1 / 2}\right) W_{j-1} / \Delta x_{j-1 / 2}, \quad j=1, \ldots, J \\
& Q_{j}=P_{j} e^{-\omega_{j}}+R_{j} e^{\omega_{j-1}}
\end{aligned}
$$

with boundary conditions

$$
P_{J}=0, \quad R_{1}=0
$$


TABLE II

Dependence of Numerical Conservation of Particles on Time-Step Size

\begin{tabular}{cc}
\hline$A \tau$ & $\Delta N(\%)$ \\
\hline 0.1 & 13.1 \\
0.01 & 2.03 \\
0.001 & 0.756 \\
\hline
\end{tabular}

and

$$
\begin{aligned}
\omega_{j} & =2 x_{j+1 / 2} \Delta x_{j+1 / 2}, \\
W_{j} & =\omega_{j} / e^{\omega_{j-1}}, \\
A\left(x_{j+1 / 2}\right) & =\frac{1}{2 x_{j+1 / 2}} \sum_{b}\left(\frac{z_{b}}{z_{T}}\right)^{2} \rho_{b}^{2} G\left(\frac{x_{j+1 / 2}}{\rho_{b}}\right) .
\end{aligned}
$$

As written, this implicit differencing scheme has the attractive properties of being both conservative and positive. The former property is achieved with suitable boundary conditions, while the latter is the result of an adaptive scheme that continuously varies the difference scheme from a centered to a forward difference.

In order to make this scheme compatible with the multigroup formalism, it is useful to make a change of index (from $j$ to $g$ where $j=1$ was the lowest energy, while $g=1$ will be the highest energy) and also to make the time differencing explicit. These changes take the form

$$
\left(\sigma_{t}^{g}+\frac{1}{\Delta \tau}\right) f_{g}^{n+1}=\sigma_{\mathrm{dow} n}^{g} \int_{g-1}^{n+1}+\sigma_{\mathrm{up}}^{g} f_{g+1}^{n}+\frac{1}{\Delta \tau} \int_{g}^{n}, \quad g=1, \ldots, G,
$$

where we have equated $\sigma_{t}^{g}$ with $Q_{g}, \sigma_{\mathrm{up}}^{g}$ with $R_{g}$, and $\sigma_{\mathrm{duwn}}^{g}$ with $\boldsymbol{P}_{g}$. Thus, we now have a velocity differencing scheme that can be utilized in a traditional multigroup format. However, the introduction of an explicit time difference leads to a relaxation of the original conservation properties of the scheme from an absolute property to one that is conditionally dependent on the "smallness" of the time step. Table II summarizes the dependence of the conservation of particles for this scheme as a function of the time-step size.

It should be noted that this can be considered to be a worst-case estimate since these values were obtained for a particle thermalization problem that involved a large degree of upscattering.

\subsection{Numerical Quadrature}

In order to monitor the conservation of particles and to calculate energy deposition, it was necessary to develop an accurate quadrature scheme in energy. A 


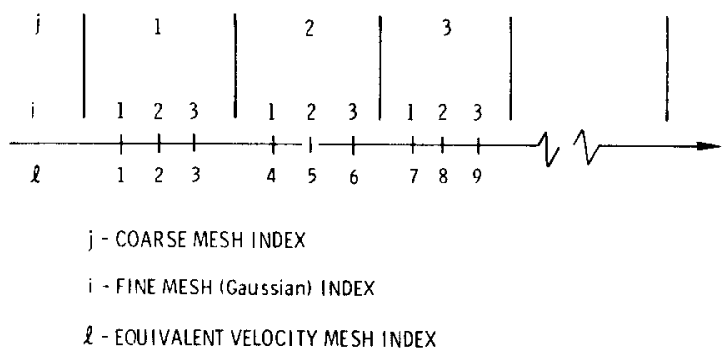

Figure 2

Gaussian quadrature scheme based on a repetitive three-point quadrature mesh (allowing for an arbitrarily large number of mesh points without restrictions due to the largest order quadrature set stored) was selected. The velocity dependent integrals have the form

$$
\begin{aligned}
\int_{a}^{b} f(x) d x & =\sum_{j=1}^{J} \int_{a+(j-1) \Delta_{j}}^{a+j \Delta_{j}} d x f(x) \\
& \cong \sum_{j=1}^{J} \frac{\Delta_{j}}{2} \sum_{i=1}^{3} \omega_{i} f\left(\frac{z_{i} \Delta_{j}+2\left(a+j \Delta_{j}\right)-\Delta_{j}}{2}\right) .
\end{aligned}
$$

The velocity mesh is shown in Fig. 2, where the equivalent mesh points are given by

$$
\begin{aligned}
\xi_{l} & =\xi_{i j}=\left(z_{i} \Delta_{j}+2\left(a+j \Delta_{j}\right)-\Delta_{j}\right) / 2, \\
l & =3(j-1)+i .
\end{aligned}
$$

Here $J$ is the total number of coarse mesh intervals, $\Delta$ is the coarse mesh spacing which is taken to be uniform and equal to $(b-a) / J$, and $\omega_{i}$ and $z_{i}$ are the Gaussian weights and quadrature points, respectively.

\subsection{Energy Deposition Calculations}

The Fokker-Planck equation for a test particle does not explicitly conserve energy. We can calculate the amount of energy that is transferred from the test particle to the field particles and its partitioning between the various background species by taking the appropriate moment of the Fokker-Planck equation and identifying the loss term as

$$
\frac{\partial \mathscr{E}(r, \tau)}{\partial \tau}=\left.\int_{0}^{\infty} x^{2} \frac{\partial f(r, x, \tau)}{\partial \tau}\right|_{\text {coll }} x^{2} d x=\int_{0}^{\infty} x^{2} \frac{\partial}{\partial x}\left\{A f+\frac{A}{2 x} \frac{\partial f}{\partial x}\right\} d x
$$


Integrating this expression by parts and working in terms of our standard integrals we find that

$\frac{\partial \mathscr{E}(r, \tau)}{\partial \tau}=\sum_{b} 2\left(\frac{z_{b}}{z_{T}}\right)^{2} \ln A_{b} \int_{0}^{\infty} x^{2} f_{T} \frac{1}{x}\left\{\left(\frac{M_{T}}{M_{b}}\right) \phi\left(\frac{x}{\rho_{b}}\right)-\left(1+\frac{M_{T}}{M_{b}}\right) \frac{2 x}{\pi^{1 / 2} \rho_{b}} e^{-x^{2} / \rho_{b}^{2}}\right\}$.

This expression is identical to those given by Sivuhkin [13] and by Trubnikov [14]. The partitioning of energy to the $b$ th background is given by each individual term in the summation.

\section{Validation OF THE Model}

As mentioned previously, the velocily-dependent and angular-dependent terms were studied separately to enable us to validate the method of numerical solution by comparison with problems that had known solutions.

\subsection{Validation of the Angular Dispersion Term-The Bethe Problem}

We have validated our treatment of the angular dispersion term by comparing TIMEX-FP results with an approximate analytic solution to the model equation

$$
\mu \frac{\partial f}{\partial \rho}=\alpha \frac{\partial}{\partial \mu}\left[\left(1-\mu^{2}\right) \frac{\partial f}{\partial \mu}\right]
$$

obtained by Bethe $[15,16]$. The dimensionless parameters are given by $\rho=z / L$ and $\alpha=L / 2 \lambda ; L$ is the slab thickness, $\lambda$ is the momentum transfer mean free path.

The problem is to determine the magnitude of the transmitted particle current through the slab geometry illustrated in Fig. 3. Bethe has obtained an approximate solution to this problem which takes the form

$$
J(L, \lambda)=\sum_{\mu_{m}>0} \frac{\omega_{m} \mu_{m} f\left(0, \mu_{m}\right)}{\left[1+\alpha\left\{\sum \omega_{m} \mu_{m} / \sum \omega_{m} \mu_{m}^{2}\right\}\right]} .
$$

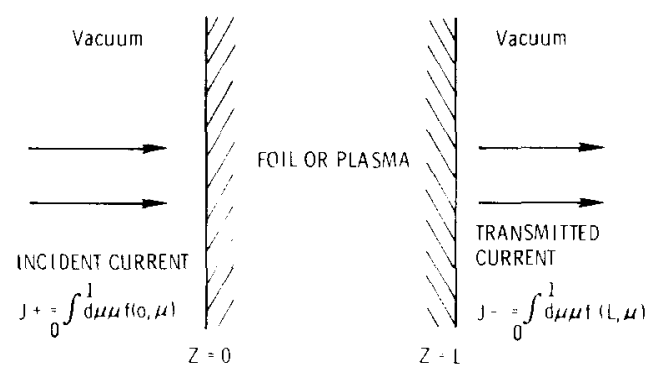

Figure 3 
TABLE III

A Comparison of the Fokker-Planck and Spencer-Lewis Methods with Respect to a Monte Carlo Standard

\begin{tabular}{ccclccccc}
\hline \multicolumn{8}{c}{ Transmitted current } \\
\hline$L$ & $\Delta x$ & $S_{N}$ & TIMEX-FP & $\begin{array}{c}\text { Spencer- } \\
\text { Lewis }^{a}\end{array}$ & $\begin{array}{c}\text { Monte } \\
\text { Carlo }\end{array}$ & $\begin{array}{c}\Delta \text { TIMEX } \\
(\%)\end{array}$ & $\begin{array}{c}\Delta \text { S-L } \\
(\%)\end{array}$ & $\begin{array}{c}\text { Source } \\
\text { incidence }\end{array}$ \\
\hline 0.5 & 0.025 & 8 & 0.56926 & 0.531 & 0.571 & 0.305 & 7.00 & Isotropic \\
1.0 & 0.050 & 8 & 0.4011051 & 0.392 & 0.413 & 2.88 & 5.08 & Isotropic \\
1.0 & 0.025 & 4 & 0.3931325 & 0.392 & 0.413 & 4.81 & 5.08 & Isotropic \\
1.0 & 0.050 & 8 & 0.4730208 & 0.471 & 0.487 & 2.87 & 3.29 & Normal \\
1.5 & 0.075 & 8 & 0.3324718 & 0.301 & 0.322 & 3.25 & 6.52 & Isotropic \\
2.0 & 0.050 & 8 & 0.260899 & 0.233 & 0.251 & 3.94 & 7.17 & Isotropic \\
2.0 & 0.050 & 4 & 0.255295 & 0.233 & 0.251 & 1.71 & 7.17 & Isotropic \\
\hline
\end{tabular}

${ }^{a}$ B. R. Wienke, K. Lee, And W. F. Miller, JR., Bull. Amer. Phys, Soc. 22 (1977), 1189.

Since we were interested in the transmitted current as given by

$$
\int_{0}^{1} d \mu \mu f(L, \mu) \cong \sum_{m=1}^{\mathrm{MM} / 2} \omega_{m} \mu_{m} f\left(L, \mu_{m}\right),
$$

that is, a half-range integration, a double- $P_{N}$ quadrature set in angle was used [17].

A comparison of results demonstrated good agreement between the computed and the approximate analytic results (which have recently been found to be quite accurate "under a wide variety of conditions" [18]) and allowed us to estimate the dependence of the computational error on the mesh spacings. It was found that the error scaled as $O(\Delta x)$ as might be expected for a linear spatial basis function. However, no quantitative error dependence on the angular mesh spacing was obtained since the transmitted current is an integral quantity and therefore the details of that dependence are smoothed out.

We have also compared our results with those reported by Weinke using the Spencer-Lewis approximation to the same problem. As can be seen in Table III, the TIMEX-FP results using a $P_{0}-S_{4}$ angular quadrature set were consistently more accurate than those given by the Spencer-Lewis approximation using a $P_{3}-S_{32}$ quadrature set. (Weinke's Monte Carlo results were used as a standard for this comparison.)

\subsection{Validation of the Velocity Differencing Scheme}

The velocity differencing scheme used in TIMEX-FP was validated by studying several simple particle equilibration problems where the equilibrium solution was known to be a Maxwellian.

Specifically, we have considered the problem of a series of test particles of mass $m$ and having an initial Maxwellian distribution at temperature $2 \mathrm{~T}$ relaxing and coming 
to thermal equilibrium with an infinite homogeneous medium composed of particles of mass $m$ at a temperature T. In these problems, 18 energy groups were employed. These problems allowed us to monitor the particle conservation as well as to check the pointwise deviation of the solution from a Maxwellian. Furthermore, we were able to verify the accuracy of the "quasi-equilibrium" solutions through which the distribution function passes by comparing the time-integrated rate of energy transfer, as given by Eq. (33), with the known difference in total energy between the initial and final distribution functions. Table II summarizes the particle conservation for this problem as a function of the time-step size. Allowing the problem to run to steady state, one finds less than a $1 \%$ maximum pointwise deviation of the solution from the true Maxwellian solution. Likewise, energy transfer can be accounted for to within $1 \%$.

\subsection{Anisotropic Velocity-Space Problems}

The differential rates of energy relaxation and angular isotropization of a distribution of test particles are dependent on the relative masses of the test and field particles (TIMEX-FP is currently capable of handling three distinct particle species, one test and two field). Winsor [19] has suggested that the full Fokker-Planck collision term physics can be demonstrated by studying the relaxation of anisotropic distributions where such differences in test and field particle masses exist. Figures 4-9 contain examples of some typical solutions obtained with TIMEX-FP for such problems.

It should be noted that one is only concerned with the details of the velocity-space physics for such problems and we have therefore implemented the study of such problems by suppressing the spatial terms in the program. Specifically, this has been achieved by restricting the spatial mesh to two mesh intervals (planar geometry) with reflecting boundaries and then placing a homogeneous initial angular flux on the spatial mesh. For this set of problems we used 18 energy points, an $S_{12}$ angular quadrature, and an initial flux having the form

$$
f(t=0)=\exp \left(-50(x-2)^{2}-10 \mu^{2}\right) .
$$

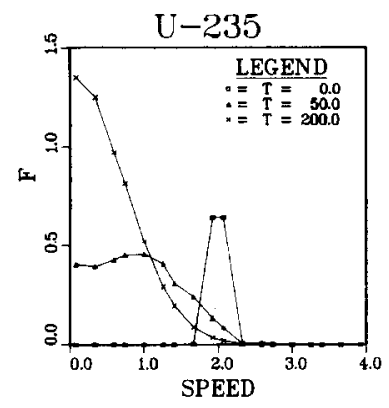

FiguRE 4

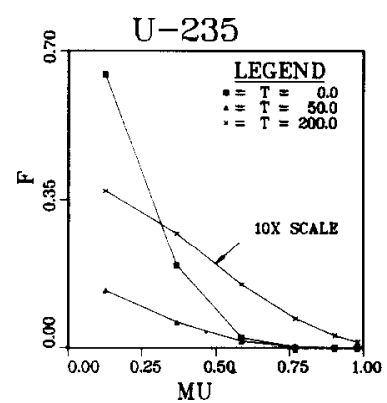

Figure 5 


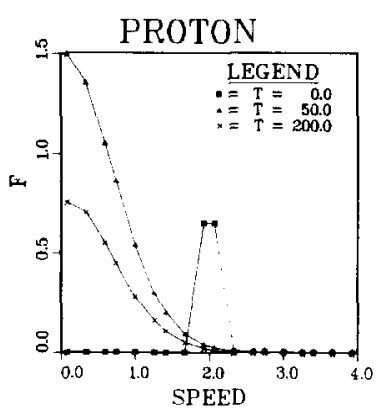

FIGURE 6

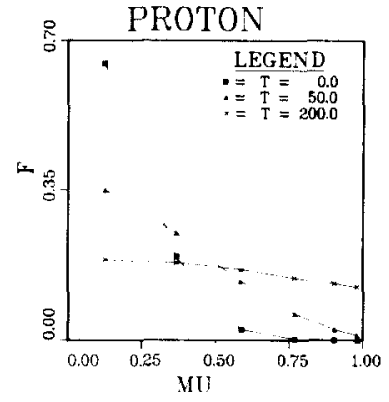

FIGURE 7

We have studied the three extremes of possible mass differences between test and field particles: (1) $U-235$ test/proton field, (2) proton test/proton field, (3) electron test/proton field.

The case of a singly ionized $U-235$ beam interacting with a proton field is presented in Figs. 4 and 5. Initially, the distribution function is peaked about $x=2.0$ and $\mu=0$ as given by Eq. (37). One can see that energy relaxation interactions dominate over angular dispersions as evidenced by the fact that the velocity distribution becomes Maxwellian while the angular distribution remains quite anisotropic. The proton-proton interaction problem is presented in Figs. 6 and 7. Starting from the same initial condition, one can see that the test particle distribution function evolves both in energy and direction to an approximately isotropic Maxwellian on the same time scale where the heavier U-235 beam still demonstrated a degree of anisotropy of the Maxwellian. For the case of a light mass electron beam interacting with a proton field (Figs. 8 and 9) we see that the distribution function is very isotropic on this same time scale; however, the beam has not experienced any significant energy relaxation. Thus, it can be seen that energy transfer collisions dominate for large mass ions interacting with a light mass field, light mass particles interacting with a heavy field experience predominantly pure elastic scattering, while like mass particles show approximately equal rates of slowing down and angular dispersion.

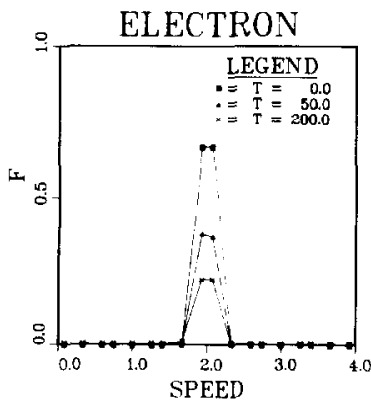

FIGURE 8

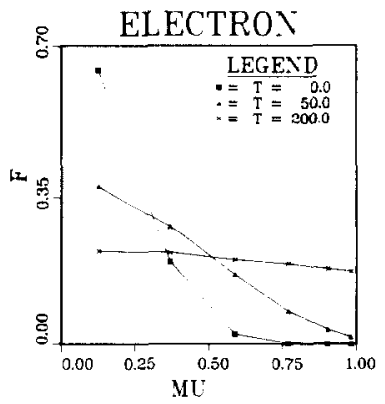

Figure 9 
It is interesting to note the correlation between the dominant physical interactions in the above problems and the numerical conservation achieved by the TIMEX-FP code for the same time-step size $(\Delta t=0.025)$. The best numerical conservation was achieved for the electron problem $(\Delta N=0.145 \%)$, the proton case was slightly worse $(\Delta N=4.48 \%)$, while the U-235 case exhibited the poorest conservation $(\Delta N=9.7 \%)$. This is related to the amount of upscattering (thermalization) that occurs in the proton and U-235 cases.

One would require additional energy grid points in the lower-energy regime or a smaller time step to achieve better conservation in these problems. (Both have the effect of minimizing the magnitude of the changes of $f$ at the lower end of the energy spectrum where the second-order thermalization effects are occurring.)

\section{Spherical Geometry Benchnark Problem}

In order to study a full phase-space problem, we consider the case of a source of $3.5-\mathrm{MeV}$ alpha particles located at the center of a uniform spherical plasma comprised of an unreacting equimolar mixture of deuterium and tritium. Determine the fraction of the alpha particle energy that is transferred to the plasma electrons and ions as a function of radius. While this problem has been studied previously by several researchers $[2-5]$, we have chosen to compare our TIMEX-FP results with the LSN results of Antal and Lee [3], since they are representative of those in the literature, and since the common heritage of the discrete ordinates and the LSN techniques make a direct comparison possible.

For a specified plasma temperature $\left(\theta_{b}=50 \mathrm{keV}\right)$, plasma density $\left(\rho=0.2125 \mathrm{~g} / \mathrm{cm}^{3}\right)$, alpha particle energy $\left(E_{0}=3.5 \mathrm{MeV}\right)$, and given particle masses, it is possible to calculate values for the reference velocity $\left(v_{0}\right)$, spatial scale length $\left(r_{0}\right)$, and temporal scale length $\left(t_{0}\right)$ used in our method. Referring to Eqs. (12) and (14), and noting that for the case of an ion test particle:

$$
\begin{gathered}
v_{0}=1.3845 \times 10^{6}\left[\frac{M_{p}}{M_{T}}\right]^{1 / 2} \theta_{b}^{1 / 2} \quad\left[\frac{\mathrm{cm}}{\mathrm{sec}}\right], \\
\left(\frac{Z_{b}}{Z_{T}}\right)^{2} \Gamma_{e}=8.06 \times 10^{7} n_{b} Z_{b}^{2} Z_{T}^{2}\left(\frac{M_{e}}{M_{T}}\right)^{2} \ln A_{e} \quad\left[\frac{\mathrm{cm}^{3}}{\mathrm{sec}^{4}}\right]
\end{gathered}
$$

and using $M_{\mathrm{DT}}=4.1815 \times 10^{-24} \mathrm{gm}$, we find that $r_{0}=2.266 \times 10^{-2} \mathrm{~cm}$ and $t_{0}=0.146 \mathrm{nsec}$. These values use an assumed value of $\ln A_{e}=8.25$.

Antal and Lee use the energy range of the alpha particles due to electron interactions as the spatial scale in discussing their results. This range is given as $\lambda_{s}=4.7 / \rho$, which for the given values of $\rho=0.2125 \mathrm{~g} / \mathrm{cm}^{3}$ yields a scale length of $\lambda_{s}-22.1177 \mathrm{~cm}$. Their spatial mesh was comprised of 20 zones of width $\Delta r=0.035 \lambda_{s}(0.7742 \mathrm{~cm})$, and they used an $S_{4}$ angular mesh with 18 energy groups.

In terms of our normalized variables, a spatial zone thickness of $0.7742 \mathrm{~cm}$ 
corresponds to a TIMEX-FP mesh spacing of $34.16 r_{0}$ which is equivalent to 20 equal thickness zones along a sphere of radius $683.2 r_{0}$. Calculations were performed using 18 energy groups, an $S_{4}$ quadrature, and a time-step size $\Delta \tau=0.1$. The energy mesh was constructed such that the alpha particle source occurred in group $g=2$ at a corresponding $x$ value of $\left(E_{0} / \theta_{b}\right)^{1 / 2}=8.366$. Using an 18-point grid with the points determined by the $n=3$ Gaussian quadrature points as discussed in Section 3.3., this corresponded to using a maximum $x$-value of 9.126 as an input to the grid generating subroutine.

The TIMEX-FP results for the cases of simple straight-line slowing-down and full Fokker-Planck collision term physics term physics (i.e., including angular dispersion) are shown in Fig. 10. As indicated, the alpha particle source was located in the first zone and was isotropically distributed on the angular mesh in the second energy group. Also presented in this figure are the results of Antal and Lee's LSN calculations. The straight-line TIMEX-FP result (no angular dispersion) is seen to be in fairly close agreement with the LSN result. It should be explicitly noted that the term "straight line," implying no angular redistribution, is used in a rigorous sense. Normally, a neutron transport version of a discrete ordinate code implicitly assumes isotropic scattering with cncrgy transfer by using the angle-integrated scalar flux in calculating the scattering contributions. However, in TIMEX-FP, this energy/angle coupling has been severed by calculating all energy transfer terms using the angledependent distribution function.

One can see that when the angular dispersion term contribution is included in the calculation, the Bragg peak shifts toward the origin due to backscattering, and increases in magnitude accordingly. For both cases, particles were conserved to within $\Delta N=4.676 \%$. As discussed in Ref. [4], the values of the electron and ion coloumb logarithms will be different for such a problem. Therefore, we have calculated and applied a correction factor of $2.75 \ln A_{e} \cong \ln A_{i}$ in this problem to give the correct ion stopping power.

It is difficult to determine whether the difference between the TIMEX-FP (straight-
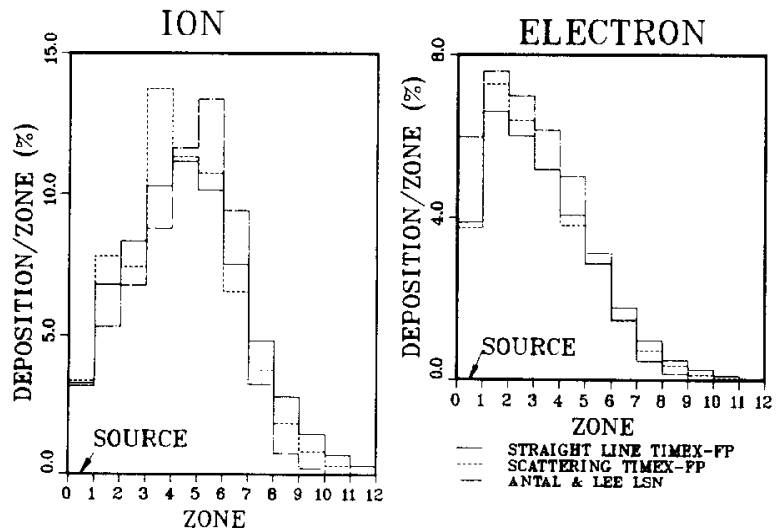

Figure 10 
line) and the LSN results are significant. The TIMEX-FP code does retain more gencral velocity-spacc physics than those used in the LSN work; however for an initial $x$ value of approximately 9.0, the continuous slowing-down approximation should be rigorous (at least for higher $x$-values). In any case, since the internal conservation checks indicate a high degree of consistency in the calculations, we feel that our results should be reliable. However, it has been suggested that the discrete ordinates method is not particularly well suited to problems involving localized sources $|4|$. It is possible that an adaptation of the first-collision source option that is often used in such localized source calculations in neutron transport problems would result in a better agreement as to the position of the Bragg peak.

It should be lastly noted that the TIMEX-FP code not only provides the timeintegrated energy deposition profile, but also provides time-dependent information on the interaction process as well as detailed information about the single-particle distribution function $f(r, \mu, v, t)$.

\section{Summary and CONCluding Remarks}

A formalism for solving the time- and space-dependent Fokker-Planck equation using the discrete ordinates method of neutron transport theory has been developed consistent with the work of Killeen and Marx. At present, this formalism has excluded the effect of electromagnetic fields, has assumed isotropic Rosenbluth potentials (i.e., an isotropic background distribution function), and further is restricted to the "test particle" case where nonlinear, self-interactions are excluded. Moreover, we have only considered the case of a Maxwellian background. The generalization of the background distribution function within the assumption of isotropy is straightforward. However, inclusion of self-interactions would require the angular dependence of the Rosenbluth potentials be retained in the collison term; although for many applications, the test particle model is a sufficiently good assumption. The formalism does retain both the angular dispersion and the velocity diffusion (thermalization) terms. Therefore, one is not restricted to the study of problems that do not include up-scattering, nor to highly collisional plasmas where an assumption of isotropy of the test particle distribution function is made. Moreover, the accuracy of the description of the energy dependence of the collision term has not been restricted either by expanding the function $G(y)$ (Eq. (10)) in the $y \gg 1$ or the $y \ll 1$ limits.

Difference relations for the Fokker-Planck collision term have been obtained and implemented in the TIMEX-FP computer code. Validation of the angular dispersion and energy transfer terms have been separately performed by studying the Bethe and the energy relaxation problems, respectively. Furthermore, the conservation of particles and energy has been documented. The combined effects of angular dispersion and energy transfer have been investigated by modeling the velocity-space relaxation of an initially anisotropic test particle distribution in an isotropic Maxwellian background. By varying the relative mass of the test and background 
particles, it has been demonstrated that energy loss dominates in the case of heavy projectiles on a light background, while angular dispersion is dominant in the light/heavy case. The two processes occur at about the same rate for like mass particles.

Finally, the space-, angle-, and energy-dependent 3.5-MeV alpha energy deposition problem has been studied. Both the position and the magnitude of the Bragg peak in the ion component are reproduced quite well for the straight-line case when compared to the representative results reported by Antal and Lee. Inclusion of angular dispersion results in a slight backscattering of the Bragg peak. Based on the result of the anisotropic relaxation problem, the inclusion of angular dispersion would be expected to have a larger effect in higher atomic number plasmas.

It should be noted that these results do not necessarily represent the most efficient scheme for utilizing the discrete ordinates method in solving the Fokker-Planck equation. Whereas we have actually modified the internal structure of the TIMEX-FP computer code, the power of the discrete ordinates method would be much more effectively utilized if one could generate "effective Fokker-Planck cross sections" that could be used in unmodified neutron transport codes. This would allow one to directly utilize all the powerful numerical techniques that have been developed over the years in neutron transport such as diffusion synthetic acceleration [20] and outer iteration rebalance $|21|$, etc. Moreover, this would obviate the need for an independent and redundant development of the large variety of geometry, boundary, and source configurations available in a large number of one- and two-dimensional discrete ordinates production codes. Studies of the possibility of naturally incorporating the angular dispersion term as an effective Legendre polynomial expanded cross section have been undertaken and may lead to such a realization as well as improvements in the speed and accuracy of the results.

\section{REFERENCES}

1. J. Killeen and K. D. Marx, in "Methods in Computational Physics" Vol. 9, Academic Press, New York, 1970.

2. E. G. Corman, W. E. Loewe, G. E. Cooper, And A. M. Winslow, Nucl. Fusion (1975), 377.

3. M. J. ANtal and C. J. Lee, J. Comput. Phys. 20 (1976), 298.

4. P. A. Haldy and J. Ligov, Nucl. Fusion 17 (1977), 1225.

5. G. A. Moses, Nucl. Sci. Eng. 64 (1977), 49.

6. J. Killeen, A. A. Mirin, And M. E. Resnick, in "Methods in Computational Physics, Vol. 16, Academic Press, New York, 1976.

7. K. D. LATHOP, Reactor Technol. 15 (1972), 107.

8. J. JunG, Nuclear Sci. Engrg. 60 (1976), 74.

9. W. H. ReEd, T. R. Hill, F. W. Brinkley, and K. D. LATHRop, LA-5428-MS, 1973.

10. T. R. Hill AND W. H. ReEd, LA-6201-MS, 1976.

11. M. N. Rosenbluth and W. M. MacDonald, Phys. Rev. 107 (1957), 1.

12. J. S. Chang and G. Cooper, J. Comput: Phys. 6 (1970), 1.

13. D. V. Sivuhkin, in "Reviews of Plasma Physics," Vol. 4, Consultants Bureau, New York, 1966.

14. B. A. Trubinkov, in "Reviews of Plasma Physics," Vol. 1, Consultants Bureau, New York, 1965. 
15. H. A. Bethe, M. E. Rose, and L. P. Smith. Proc. Amer. Philos. Soc, 78 (1938), 573.

16. T. A. MehlhorN. Ph.D. dissertation. The University of Michigan, 1978.

17. G. I. Bell and S. Glasstone, "Nuclear Reactor Theory," pp. 220-226. (Van Nostrand, Princeton, N. J.. 1970.

18. D. Stein and I. B. Bernstein, Phys. Fluids 19 (1976), 811.

19. N. K. Winsor. Nucl. Sci. Eng. 64 (1977), 33.

20. R. E. Alcouffe, Nucl. Sci. Eng. 64 (1977), 344.

21. T. R. HILL, LA-5990-MS, 1975. 